\title{
TITLE:
}

\section{Utilization of direct smears of thyroid fine-needle aspirates for ancillary molecular testing: a comparison of two proprietary testing platforms}

\author{
Kristen L. Partyka, M.D., ${ }^{1}$ Melissa L. Randolph, B.S., SCT(ASCP), ${ }^{1}$ Karen A. Lawrence, B.S., MLT, \\ CT(ASCP), ${ }^{1}$ Harvey Cramer, M.D., ${ }^{1}$ and Howard H. Wu, M.D. ${ }^{1}$
}

\author{
${ }^{1}$ Department of Pathology and Laboratory Medicine \\ Indiana University School of Medicine \\ 350 W. 11th Street \\ Indianapolis, IN 46202 \\ United States of America \\ Email: partykak@iupui.edu and hhwu@iupui.edu
}

RUNNING TITLE: Thyroid FNA direct smears and molecular testing

\author{
Corresponding author: \\ Kristen L. Partyka, MD \\ Indiana University School of Medicine \\ IU Health Pathology Laboratory \\ 350 W. 11th Street, IUHPL \#4086 \\ Indianapolis, IN 46202 \\ Telephone: $\quad 317-491-6154$ \\ Facsimile: $\quad$ 317-491-6419 \\ Email: partykak@iupui.edu \\ Total number of text pages: 13; tables: 4
}

Disclosures: There are no prior publications or submissions with any overlapping information, including studies and patients. This manuscript has not been and will not be submitted to any other journal while it is under consideration. Each author listed on the manuscript has seen and approved the submission of this version of the manuscript and takes full responsibility for the manuscript. The authors have no financial or conflicts of interest to disclose. This research received no specific grant from any funding agency in the public, commercial, or not-for-profit sectors.

This is the author's manuscript of the article published in final edited form as:

Partyka, K. L., Randolph, M. L., Lawrence, K. A., Cramer, H., \& Wu, H. H. (2018). Utilization of direct smears of thyroid fineneedle aspirates for ancillary molecular testing: A comparison of two proprietary testing platforms. Diagnostic Cytopathology, 46(4), 320-325. https://doi.org/10.1002/dc.23902 


\section{ABSTRACT: \\ BACKGROUND:}

Ancillary molecular testing has been recommended for thyroid fine-needle aspirates (FNA) with indeterminate cytologic diagnoses. Rosetta Genomics and Interpace Diagnostics have developed assays that can utilize direct smears as the testing substrate.

\section{METHODS:}

A retrospective study of indeterminate thyroid FNAs with known histologic follow-up was performed. One Diff-Quik-stained smear and one Papanicolaou-stained smear with similar cellularity (at least 60-100 lesional cells) from each case were sent to Rosetta and Interpace, respectively, for analysis. The results were directly compared and correlated with the final histopathology. Neither company was aware of the follow-up histologic findings in these cases.

\section{RESULTS:}

A total of 10 thyroid FNAs were identified from our 2015 files. The cytologic diagnoses included follicular lesion of undetermined significance (FLUS, $n=5$ ), follicular neoplasm/suspicious for follicular neoplasm (FN/SFN, $n=4)$, and suspicious for malignancy (SM, $n=1)$. Of the seven cases with benign histology, 6 smears were classified as benign by the RosettaGX ${ }^{\mathrm{TM}}$ microRNA classifier, and 1 case was designated as suspicious. 5 cases were negative by both ThyGenX ${ }^{\mathrm{TM}}$ oncogene panel and ThyraMIR ${ }^{\mathrm{TM}}$ microRNA classifier. 1 case was negative by ThyGenX and positive on follow-up ThyraMIR, and 1 case was positive for KRAS mutation and positive on ThyraMIR. Both the RosettaGX and ThyGenX/ThyraMIR tests demonstrated positive results for the 3 histologically malignant cases.

\section{CONCLUSION:}

This study demonstrates that two molecular testing platforms performed equally well using our stained direct smears. Both molecular tests revealed a 100\% negative predictive rate. RosettaGX showed a $75 \%$ positive predictive value (PPV) in comparison to $60 \%$ for ThyGenX/ThyraMIR.

KEYWORDS: thyroid cytology; FNA; the Bethesda System; molecular testing; direct smears 


\section{MANUSCRIPT TEXT:}

\section{INTRODUCTION:}

Thyroid cancer is the most common endocrine malignancy, and its incidence has steadily increased over the past few decades. It may present as a nodule palpated on physical exam or as an incidental finding on imaging. ${ }^{1-2}$ As with any mass or nodule detected, there is a fundamental level of uncertainty regarding its diagnosis and prognosis. Fine-needle aspiration (FNA) plays an important role in the initial evaluation of these patients. ${ }^{3}$ With its high sensitivity and specificity, it provides vital information for clinicians to formulate treatment plans and decide which patients might benefit from surgery. ${ }^{4}$

The Bethesda System for Reporting Thyroid Cytopathology (TBSRTC) recommends separation of FNAs into six main categories based on defined criteria. Each diagnostic group denotes a certain risk of malignancy. ${ }^{4-5}$ The majority of aspirates can diagnose thyroid nodules as benign or malignant. ${ }^{2,6-8}$ However, a major limitation to thyroid FNA is the equivocal nature of numerous smears. ${ }^{7}$ The subjective aspect of interpretation and wide interobserver variability add additional levels of complexity. ${ }^{3-4,9} \mathrm{Up}$ to $30-40 \%$ of cases are borderline and classified as indeterminate. ${ }^{2,6,8-10}$ They are incorporated into the following groups: atypia of undetermined significance/follicular lesion of undetermined significance (AUS/FLUS, Bethesda category III); follicular neoplasm/suspicious for follicular neoplasm (FN/SFN, Bethesda category IV); and suspicious for malignancy (SM, Bethesda category V). ${ }^{5}$

The study of molecular genetics in disease is a rapidly expanding field. Progress with research has identified genetic alterations associated with thyroid cancer. ${ }^{2,4,11}$ It has created opportunities to increase the diagnostic accuracy of thyroid FNA and redefine indeterminate aspirates for clinical management. ${ }^{4,12}$ The importance of molecular markers was recently addressed in the 2015 American Thyroid Association (ATA) guidelines. Ancillary molecular testing has been recommended for thyroid fine-needle aspirates with indeterminate cytologic diagnoses., ${ }^{4,8-9,13}$ 
Many thyroid molecular tests employed by clinicians (Afirma and ThyroSeq) require that additional aspirates be procured and submitted in special collection media., ${ }^{4,6}, 14$ Afirma Gene Expression Classifier (GEC) from Veracyte (South San Francisco, CA) uses microarray technology to assess the mRNA expression profile of indeterminate thyroid aspirates. It requires that personnel attain two dedicated FNA passes for evaluation with their proprietary algorithm. In comparison, ThyroSeq v2 (CBLPath, Rye Brook, NY) utilizes next-generation sequencing techniques to examine DNA mutations and RNA fusions. It has the particular requirement of needing 1-2 drops of aspirate material from the first pass of a thyroid FNA (Table 1). ${ }^{4,9}$

However, two companies, Rosetta Genomics and Interpace Diagnostics, have developed microRNAbased assays that can utilize direct smears as the testing substrate. ${ }^{1,6}$ Advancements with next-generation sequencing have allowed the discovery of novel targets for molecular testing, including microRNAs (miRNAs). ${ }^{10}$ MicroRNAs are small, noncoding RNA molecules that are intricately involved with regulating cellular function. They play a role in controlling the cell cycle with gene expression, proliferation, and survival. ${ }^{1,4,8,15}$ Numerous publications have reported the divergent expression of miRNAs in distinct tumors, including thyroid cancer. ${ }^{1,6,10,12,15}$

Rosetta GX Reveal is a relatively new microRNA-based assay, and few articles have been published in the literature. ${ }^{1,6}$ This test evaluates the expression of 24 miRNAs, including not only ones upregulated in papillary thyroid carcinoma but also those that are downregulated. It has the unique feature of being developed for use with routinely prepared cytology smears (Table 1 ). ${ }^{1}$ The concept of extracting miRNAs from Papanicolaou or Romanowsky-stained slides is cutting edge for thyroid cytopathology. ${ }^{15}$

Furthermore, Interpace Diagnostics introduced a multiplatform-testing algorithm to aid in the evaluation of indeterminate aspirates. ThyGenX is based on the oncogene panel originally marketed as miRInform by Asuragen. ${ }^{4,7}$ It maintains the same 4 DNA point mutations and 3 RNA translocation fusion markers 
that are highly specific for differentiated thyroid cancer. ${ }^{10,12}$ It has been modified with next-generation sequencing and now can detect more than 100 genetic alterations across 8 genes (Table 1). ${ }^{4}$ Moreover, ThyraMIR is a molecular test designed to analyze the expression of 10 different microRNAs to further modify the risk of malignancy. ${ }^{4,8,10,15}$

In the two-step process at Interpace, ThyGenX is performed first. If this oncogene panel is negative or only RAS mutations are identified, a reflex ThyraMIR test is then performed. ${ }^{9-10}$ With its initial release, ThyGenX and ThyraMIR required one additional FNA pass with at least 50 ng of cellular material submitted in a special preservative solution. Many recent review articles comparing the contemporary testing options available for thyroid aspirates perpetuate this factor. ${ }^{4,9}$ However, ThyGenX and ThyraMIR have been successfully performed on conventionally stained cytology smears, monolayer Thin Prep slides, and even formalin-fixed paraffin-embedded tissue through their internal validation process (Table 1). Our study is the first to examine the feasibility of using the direct smears as the test samples.

Utilizing direct smears for molecular testing is an exciting advancement in the field of thyroid cytology. With any FNA, there is a risk that the final interpretation may deviate from the rapid assessment. If nothing suspicious is appreciated at the on-site evaluation, cytologists may not request additional material up front. The ability to employ direct smears holds great promise for personalized medicine. Molecular assays can potentially be performed on prior cases in the archives to further aid treatment plans. It creates additional options, more flexibility, and a larger window of opportunity for its application in the clinical setting.

\section{MATERIALS AND METHODS:}

This study was approved by the Institutional Review Board of Indiana University. A retrospective analysis of indeterminate thyroid FNAs with known histologic follow-up in 2015 was conducted at our 
institution. A computer search of the cytopathology archives was performed to identify indeterminate aspirates with material collected for Afirma GEC testing and subsequent surgical intervention. All aspirates were categorized using the Bethesda System for Reporting Thyroid Cytopathology (TBSRTC). The cases had been evaluated by different board-certified cytopathologists and often presented at a consensus conference.

We sought to evaluate the use of routinely stained cytology smears with two proprietary molecular testing platforms. Experienced cytotechnologists reviewed the cases to assess the level of cellularity on each smear. Rosetta accepts either air-dried Diff-Quik-stained or alcohol-fixed Papanicolaou-stained smears while Interpace only accepts Papanicolaou-stained smears. Therefore, one Diff-Quik-stained smear and one Papanicolaou-stained smear with similar cellularity (at least 60-100 lesional cells) were selected from each of 10 cases. The Diff-Quik-stained slide was sent to Rosetta Genomics (Rehovot, Israel), and the Papanicolaou-stained slide was submitted to Interpace Diagnostics (Parsippany, New Jersey) for molecular testing.

The slides were stored and shipped at room temperature. Before performing the respective analyses, both Rosetta and Interpace take high-resolution images of the slides in their entirety. The whole slide images are archived and can be provided to the clients upon future request. The results were directly compared and correlated with the final histopathology. Neither company was aware of the follow-up histologic findings in these cases.

\section{RESULTS:}

A total of 10 thyroid FNAs (7 females, 3 males; ages 25-65) were identified from our 2015 files. The cytologic diagnoses included follicular lesion of undetermined significance (FLUS, n=5), follicular neoplasm/suspicious for follicular neoplasm (FN/SFN, n=4), and suspicious for malignancy (SM, n=1). 
All cases had material collected for Afirma GEC at the time of rapid assessment, and the test was performed in 7/10 of the samples (Table 2).

Seven patients (7/10) had a total thyroidectomy, and three (3/10) underwent a lobectomy (Table 2). Investigating the circumstances surrounding the clinical decision to operate was beyond the scope of this study. The histologic results included nodular hyperplasia $(n=1)$ and follicular adenoma $(n=6)$. Malignancies comprised follicular variant of papillary thyroid carcinoma (FVPTC, $n=1$ ), papillary thyroid carcinoma (PTC, $n=1)$, and follicular carcinoma (n=1) (Table 3).

All 10 smears passed the preanalytical steps for quality assurance and were evaluated for microRNA (miRNA) expression with RosettaGX Reveal. Also, each case was interrogated for gene mutations and rearrangements with the oncogene panel ThyGenX. Only 9/10 of the cases were reflexed to the second tier of testing to detect miRNAs with ThyraMIR (Table 3).

Of the seven cases with benign histology, 6 FNA smears were classified as benign by RosettaGX, and 1 case was designated as suspicious. 5 cases were classified as negative by both ThyGenX and ThyraMIR. 1 case was negative by ThyGenX and positive on follow-up ThyraMIR with a 50-60\% risk of malignancy, and 1 case was positive for KRAS mutation and positive on ThyraMIR with a 50\% risk of malignancy. In six of the benign cases, Afirma had been performed prior to our analysis. Only two were classified as benign while 4 of them were designated as suspicious (Table 3).

Both the RosettaGX and ThyGenX/ThyraMIR tests demonstrated positive results for the 3 histologically malignant cases. Afirma GEC was performed in one malignant case and also demonstrated a suspicious result (Table 3). 


\section{DISCUSSION:}

Thyroid nodules are prevalent in the general population. Many are palpable on clinical exam while others are incidental findings on imaging. Fine-needle aspiration has proven to be a safe, minimally invasive, and cost-effective method for assessing these nodules. With each update in management guidelines published by the ATA, it continues to remain an integral part of the initial diagnostic evaluation. ${ }^{4}$ Most thyroid FNAs reliably identify samples as benign or malignant, and this can aid in directing treatment. ${ }^{6}$

However, a compelling number are classified as indeterminate according to TBSRTC. The aggregate values range anywhere from $15-30 \%$ to $10-40 \%$ in the literature. ${ }^{1,6,9-10}$ The implied probability of malignancy within these indeterminate categories creates challenges for clinicians. According to TBSRTC, the risk of malignancy is 5-15\% for AUS/FLUS (III), 15-30\% for FN/SFN (IV), and 60-75\% for SM (V). ${ }^{4-6}$ These numbers are too high to simply promote watchful waiting and follow-up. ${ }^{3-4,9}$

Without clearer management guidelines, diagnostic lobectomy is a common surgical approach in treatment of patients with indeterminate diagnosis on FNA. ${ }^{9}$ The majority of these nodules, ranging from 60-70\%, prove to be benign on final histopathology, and surgical resection might be considered overtreatment. ${ }^{2,4,9-10}$ In contrast, those cases that prove malignant on lobectomy likely subject the patient to additional treatment. This might include a completion thyroidectomy or radioactive iodine ablation with subsequent risk of complications..$^{2-3,9}$

Improving the diagnostic yield from thyroid FNA is at the forefront of current research in cytopathology.,12 Close analysis of nuclear detail and cytomorphologic characteristics will never completely eliminate the indeterminate category., Application of immunocytochemical stains also proved inadequate with low specificity and minimal reproducibility. ${ }^{4,7}$ As with many areas in medicine, the focus has shifted to molecular testing and detection of genetic alterations in thyroid cancer. ${ }^{2-4,12}$ The 
goal is to increase the number of malignancies accurately diagnosed on FNA and to reduce the extent of potentially avoidable surgeries. ${ }^{1,6-7,9-10}$

The 2015 ATA management guidelines propose the use of molecular studies in indeterminate aspirates to enhance risk stratification and treatment planning. ${ }^{2,4,8,12-13}$ Much of the research surrounds identification of BRAF and RAS point mutations and RET/PTC and PAX8/PPAR $\gamma$ rearrangements. ${ }^{6,9,12,14}$ These four genes are well known to be the most common in differentiated thyroid cancer and have implications for diagnosis and prognosis. ${ }^{4,711}$ Nevertheless, there is a subset of tumors that lack these genetic aberrations. ${ }^{10}$

Improvements in next-generation sequencing have substantially broadened the number of biomarkers that can be queried in a single thyroid aspirate..$^{4,10,15}$ Since its commercial availability in 2011, Afirma GEC has made a significant impact on thyroid cytology. It uses a proprietary algorithm to analyze mRNA expression levels of 167 genes (Table 1). ${ }^{4,8}$ Its high negative predictive value and utility as a "rule out" test have been widely advertised among cytopathologists, endocrinologists, and surgeons. ${ }^{4,9-10} \mathrm{~A}$ drawback to the test is that it calls for two dedicated FNA passes. The decision to collect for Afirma GEC has to be made at the time of rapid assessment. Unless aspirates are immediately stored in preservative and shipped under specific conditions, the test cannot be performed. ${ }^{4,14}$

Rosetta Genomics and Interpace Diagnostics are two companies that have recently developed microRNAbased assays that can be successfully performed on direct smears. Preliminary data from the multicenter validation studies of Rosetta GX Reveal miRNA classifier are encouraging. The type of stain does not affect the assay, and it can be performed on smears that are stained with Papanicolaou, Diff-Quik, or Giemsa methods. ${ }^{1,6}$ Data collected from internal validation with ThyGenX/ThyraMIR (Interpace) also show good results, but the literature does not address their use with routinely prepared smears. ${ }^{4,6,14}$ 
The specifications from the manufacturers were naturally considered when designing our study. Preanalytic factors can never be completely excluded from having an impact on the data collection. In accordance with analytic validation studies, Rosetta Genomics allows submission of either Diff-Quikstained or Papanicolaou-stained slides. In contrast, Interpace can only accept Papanicolaou-stained smears. We sought to minimize the influence from preanalytic variables by carefully selecting slides of comparable cellularity from each case. One air-dried Diff-Quik-stained slide was shipped to Rosetta, and one Papanicolaou-stained smear was sent to Interpace.

As with other molecular platforms, it is important to recognize that RosettaGX and ThyGenX/ThyraMIR are send-out tests. Diagnostic performance is one of many factors that will affect their utility with the advent of personalized medicine. Rosetta provides a rapid turnaround time of 7 days, and Interpace (ThyGenX/ThyraMIR) delivers a report within 10 to 14 days. Admittedly, conducting these assays will incur an extra cost for healthcare. Both companies work with patients, insurance plans, and various federal agencies to negotiate that aspect. The exact cost per test is likely dependent on many variables. A thorough examination may be of value in the future.

This study demonstrates that these two molecular testing platforms performed equally well using our stained direct smears. Similar to Afirma GEC, the results from Rosetta are released as either benign or suspicious. RosettaGX had one false positive in our sample population. The smear was flagged as suspicious, and it was associated with a follicular adenoma on subsequent thyroidectomy.

In contrast, Interpace uses a two-step algorithm for testing and takes a different approach with their results. If ThyGenX oncogene panel is positive, it characterizes the mutation and assigns an associated risk of malignancy. When ThyGenX is negative or only RAS mutations are identified, reflex ThyraMIR is performed. The risk of malignancy is subsequently modified based on the microRNA expression. ${ }^{9-10}$ The 
established system does not separate samples into clearcut benign or malignant categories. It was developed to provide additional data for the clinical team to formulate treatment plans.

As with many ancillary tests, the results should be considered along with patient history, presenting symptoms, and imaging characteristics. Risk assessment does not directly conform to traditional calculations of sensitivity and specificity. To foster some statistical analysis of our results, we used the interpretation of $50 \%$ or higher risk of malignancy as the cutoff for a positive test. ThyGenX/ThyraMIR resulted in two false positives, and both correlated with follicular adenomas on resection. One was negative on ThyGenX and positive on reflex ThyraMIR with an estimated 50-60\% risk of malignancy. The second case was positive for KRAS mutation (ThyGenX) and ThyraMIR with a projected risk of $50 \%$ (Table 3).

When assessing risk of malignancy, it is evident that our results are based on a low number of cases, and this may bias the figures. We acknowledge this as a potential limitation, but we hope our findings can spark interest for future investigation. Both RosettaGX and ThyGenX/ThyraMIR demonstrated a sensitivity of $100 \%$. This is along the same trajectory as the data described in the literature with a sensitivity of $85 \%$ for RosettaGX and $89 \%$ for ThyGenX/ThyraMIR. ${ }^{1,49-10}$ In our analysis, RosettaGX demonstrated a specificity of $86 \%$, which is higher than the industry reported value of $72 \% .{ }^{1}$ In comparison, ThyGenX/ThyraMIR showed a specificity of 71\%, which is slightly lower than the published value of $85 \%$ (Table 4$){ }^{4,9-10}$

Both molecular tests revealed a 100\% negative predictive rate. RosettaGX showed a $75 \%$ positive predictive value (PPV) in comparison to $60 \%$ for ThyGenX/ThyraMIR (Table 4). The data is undoubtedly affected by our small sample size, but it shows promising results. Utilizing stained direct smears is a new and innovative approach to molecular analysis in thyroid cytology. A long-term analysis with RosettaGX and multiplatform ThyGenX/ThyraMIR on a larger sample size is needed to reinforce the strength of 
these assays with direct smears. Even if additional material is not requested during the on-site evaluation, there are now more options available for ancillary testing.

\section{REFERENCES:}

1. Lithwick-Yanai G, Dromi N, Shtabsky A, et al. Multicentre validation of a microRNA-based assay for diagnosing indeterminate thyroid nodules utilising fine needle aspirate smears. J Clin Pathol. 2016; 0: 1-8.

2. Smith RB, Ferris RL. Utility of diagnostic molecular markers for evaluation of indeterminate thyroid nodules. JAMA Otolaryngol Head \& Neck Surg. 2016; 142: 421-22.

3. Cibas ES, Baloch ZW, Fellegara G, et al. A prospective assessment defining the limitations of thyroid nodule pathologic evaluation. Ann Intern Med. 2013; 159: 325-32.

4. Zhang M, Lin O. Molecular testing of thyroid nodules: A review of current available tests for fineneedle aspiration specimens. Arch Pathol Lab Med. 2016; 140: 1338-44.

5. Cibas ES, Ali SZ. The Bethesda System for Reporting Thyroid Cytopathology. Am J Clin Pathol. 2009; 132: 658-665.

6. Benjamin H, Schnitzer-Perlman T, Shtabsky A, et al. Analytical validity of a microRNA-based assay for diagnosing indeterminate thyroid FNA smears from routinely prepared cytology slides. Cancer Cytopathol. 2016; 124: 711-21. 
7. Rossi ED, Bizzaro T, Schmitt FC. Utilization of molecular testing in thyroid cytology: A concrete aid? New insights. Pathology Case Reviews. 2015; 20: 129-32.

8. Onenerk AM, Pusztaszeri MP, Canberk S, Faquin WC. Triage of the indeterminate thyroid aspirate: What are the options for the practicing cytopathologist? Cancer Cytopathol. 2017; 125 (6 suppl): 47785.

9. Nishino M. Molecular cytopathology for thyroid nodules: A review of methodology and test performance. Cancer Cytopathol. 2016; 124: 14-27.

10. Labourier E, Shifrin A, Busseniers AE, et al. Molecular testing for miRNA, mRNA, and DNA on fine-needle aspiration improves the preoperative diagnosis of thyroid nodules with indeterminate cytology. J Clin Endocrinol Metab. 2015; 100: 2743-2750.

11. Giordano TJ. Follicular cell thyroid neoplasia: insights from genomics and The Cancer Genome Atlas research network. Curr Opin Oncol. 2016; 28: 1-4.

12. Nikiforov YE. Molecular diagnostics of thyroid tumors. Arch Pathol Lab Med. 2011; 135: 569-577.

13. Haugen BR, Alexander EK, Bible KC, et al. 2015 American Thyroid Association management guidelines for adult patients with thyroid nodules and differentiated thyroid cancer: the American Thyroid Association guidelines task force on thyroid nodules and differentiated thyroid cancer. Thyroid. 2016; 26: 1-133.

14. VanderLaan PA. Molecular markers: Implications for cytopathology and specimen collection. Cancer Cytopathol. 2015; 123: 454-60. 
15. Beca F, Schmitt F. MicroRNA signatures in cytopathology: Are they ready for prime time? Cancer Cytopathol. 2016; 613-15. 